\title{
OPS-QFTs: A new type of quaternion Fourier transforms based on the orthogonal planes split with one or two general pure quaternions
}

\author{
Eckhard Hitzer \\ Department of Applied Physics, University of Fukui, 910-8507 Japan
}

\begin{abstract}
We explain the orthogonal planes split (OPS) of quaternions based on the arbitrary choice of one or two linearly independent pure unit quaternions $f, g$. Next we systematically generalize the quaternionic Fourier transform (QFT) applied to quaternion fields to conform with the OPS determined by $f, g$, or by only one pure unit quaternion $f$, comment on their geometric meaning, and establish inverse transformations.
\end{abstract}

Keywords: Clifford geometric algebra, quaternion geometry, quaternion Fourier transform, inverse Fourier transform, orthogonal planes split

PACS: AMS Subj. Class. 15A66, 42A38

References [2, 3, 4, 5] give background on quaternion Fourier transformations. For details and proofs of the orthogonal planes split we refer the reader to [6].

Gauss, Rodrigues and Hamilton's four-dimensional (4D) quaternion algebra $\mathbb{H}$ is defined over $\mathbb{R}$ with three imaginary units:

$$
i j=-j i=k, j k=-k j=i, k i=-i k=j, i^{2}=j^{2}=k^{2}=i j k=-1 .
$$

Every quaternion can be written explicitly as

$$
q=q_{r}+q_{i} i+q_{j} j+q_{k} k \in \mathbb{H}, \quad q_{r}, q_{i}, q_{j}, q_{k} \in \mathbb{R},
$$

and has a quaternion conjugate (equivalent to reversion in $\mathrm{Cl}_{3,0}^{+}$)

$$
\tilde{q}=q_{r}-q_{i} i-q_{j} j-q_{k} k, \quad \widetilde{p q}=\tilde{q} \tilde{p},
$$

which leaves the scalar part $q_{r}$ unchanged. This leads to the norm of $q \in \mathbb{H}$

$$
|q|=\sqrt{q \tilde{q}}=\sqrt{q_{r}^{2}+q_{i}^{2}+q_{j}^{2}+q_{k}^{2}}, \quad|p q|=|p||q| .
$$

The part $\boldsymbol{q}=q-q_{r}=(q-\tilde{q}) / 2=q_{i} i+q_{j} j+q_{k} k$ is called a pure quaternion, and it squares to the negative number $-\left(q_{i}^{2}+q_{j}^{2}+q_{k}^{2}\right)$. Every unit quaternion (i.e. $|q|=1$ ) can be written as

$$
\begin{aligned}
& q=q_{r}+q_{i} i+q_{j} j+q_{k} k=q_{r}+\sqrt{q_{i}^{2}+q_{r}^{2}+q_{k}^{2}} \hat{\boldsymbol{q}}=\cos \alpha+\sin \alpha \hat{\boldsymbol{q}}=e^{\alpha \hat{\boldsymbol{q}}} \\
& \cos \alpha=q_{r}, \quad \sin \alpha=\sqrt{q_{i}^{2}+q_{r}^{2}+q_{k}^{2}}, \quad \hat{\boldsymbol{q}}=\frac{q_{i} i+q_{j} j+q_{k} k}{\sqrt{q_{i}^{2}+q_{r}^{2}+q_{k}^{2}}}, \quad \hat{\boldsymbol{q}}^{2}=-1 .
\end{aligned}
$$

The inverse of a non-zero quaternion is

$$
q^{-1}=\frac{\tilde{q}}{|q|^{2}}=\frac{\tilde{q}}{q \tilde{q}} .
$$

The symmetric scalar part of a quaternion is defined as

$$
S c(q)=q_{r}=\frac{1}{2}(q+\tilde{q}), \quad S c(p q)=S c(q p)=p_{r} q_{r}-p_{i} q_{i}-p_{j} q_{j}-p_{k} q_{k},
$$


with linearity

$$
S c(\alpha p+\beta q)=\alpha S c(p)+\beta S c(q)=\alpha p_{r}+\beta q_{r}, \quad \forall p, q \in \mathbb{H}, \alpha, \beta \in \mathbb{R} .
$$

The scalar part and the quaternion conjugate allow the definition of the $\mathbb{R}^{4}$ inner product of two quaternions $p, q$ as

$$
S c(p \widetilde{q})=p_{r} q_{r}+p_{i} q_{i}+p_{j} q_{j}+p_{k} q_{k} \in \mathbb{R} .
$$

We consider an arbitrary pair of linearly independent nonorthogonal pure quaternions $f, g, f^{2}=g^{2}=-1, f \neq \pm g$. The orthogonal 2D planes split (OPS) is then defined with respect to the linearly independent pure unit quaternions $f, g$ as

$$
q_{ \pm}=\frac{1}{2}(q \pm f q g)
$$

We thus observe, that $f q g=q_{+}-q_{-}$, i.e. under the map $f() g$ the $q_{+}$part is invariant, but the $q_{-}$part changes sign.

Both parts are two-dimensional, and span two completely orthogonal planes. The $q_{+}$plane is spanned by the orthogonal quaternions $\{f-g, 1+f g\}$ whereas the $q$ - plane is e.g. spanned by $\{f+g, 1-f g\}$.

Lemma 1 (Orthogonality of two OPS planes). Given two quaternions $q$, $p$ and applying the OPS with respect to two linearly independent pure unit quaternions $f, g$ we get zero for the scalar part of the mixed products

$$
S c\left(p_{+} \widetilde{q}_{-}\right)=0, \quad S c\left(p_{-} \widetilde{q}_{+}\right)=0 .
$$

The set $\{f-g, 1+f g, f+g, 1-f g\}$ forms an orthogonal basis of $\mathbb{H}$ interpreted as $\mathbb{R}^{4}$. We can therefore use the following representation for every $q \in \mathbb{H}$ by means of four real coefficients $q_{1}, q_{2}, q_{3}, q_{4} \in \mathbb{R}$

$$
\begin{gathered}
q=q_{1}(1+f g)+q_{2}(f-g)+q_{3}(1-f g)+q_{4}(f+g), \\
q_{1}=S c\left(q(1+f g)^{-1}\right), \quad q_{2}=S c\left(q(f-g)^{-1}\right), \quad q_{3}=S c\left(q(1-f g)^{-1}\right), \quad q_{4}=S c\left(q(f+g)^{-1}\right) .
\end{gathered}
$$

As an example we have for $f=i, g=j$ we obtain

$$
q_{1}=\frac{1}{2}\left(q_{r}+q_{k}\right), \quad q_{2}=\frac{1}{2}\left(q_{i}-q_{j}\right), \quad q_{3}=\frac{1}{2}\left(q_{r}-q_{k}\right), \quad q_{4}=\frac{1}{2}\left(q_{i}+q_{j}\right) .
$$

Theorem 1 (Determination of $f, g$ from given analysis planes). Assume that two desired analysis planes are given by a set of four orthogonal quaternions $\{a, b\}$ and $\{c, d\}$. Without restriction of generality $a^{2}=c^{2}=-1$.

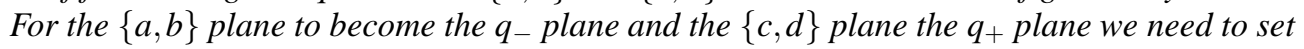

$$
f=b a, \quad g=S c(f \tilde{a}) a-S c(f \tilde{c}) c .
$$

For the opposite assignment of the $\{a, b\}$ plane to become the $q_{+}$plane and the $\{c, d\}$ plane the $q_{-}$plane, we only need to change the sign of $g$, i.e. we can set

$$
f=b a, \quad g=S c(f \tilde{c}) c-S c(f \tilde{a}) a .
$$

The map $f() g$ rotates the $q_{-}$plane by $180^{\circ}$ around the $q_{+}$axis plane. This interpretation of the map $f() g$ is in perfect agreement with Coxeter's notion of half-turn in [1].

The following identities hold

$$
e^{\alpha f} q_{ \pm} e^{\beta g}=q_{ \pm} e^{(\beta \mp \alpha) g}=e^{(\alpha \mp \beta) f} q_{ \pm} .
$$

The general double sided orthogonal planes (i.e. 2D subspaces) split quaternion Fourier transform (OPS-QFT) is defined as

$$
\mathscr{F}^{f, g}\{h\}(\omega)=\int_{\mathbb{R}^{2}} e^{-f x_{1} \omega_{1}} h(\boldsymbol{x}) e^{-g x_{2} \omega_{2}} d^{2} \boldsymbol{x},
$$

where $h \in L^{1}\left(\mathbb{R}^{2}, \mathbb{H}\right), d^{2} \boldsymbol{x}=d x_{1} d x_{2}$ and $\boldsymbol{x}, \omega \in \mathbb{R}^{2}$. The OPS-QFT (17) is invertible

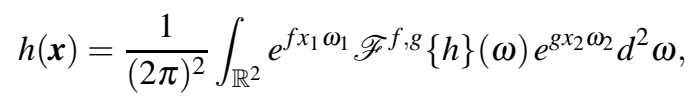


Linearity of the integral allows us to use the OPS split $h=h_{-}+h_{+}, h_{ \pm}=\frac{1}{2}(h \pm f h g)$

$$
\mathscr{F}^{f, g}\{h\}(\omega)=\mathscr{F}^{f, g}\left\{h_{-}\right\}(\omega)+\mathscr{F}^{f, g}\left\{h_{+}\right\}(\omega)=\mathscr{F}_{-}^{f, g}\{h\}(\omega)+\mathscr{F}_{+}^{f, g}\{h\}(\omega),
$$

since by its construction the operators of the Fourier transformation $\mathscr{F} f, g$, and of the OPS with respect to $f, g$ commute. From (16) follows

Theorem 2 (OPS-QFT of $h_{ \pm}$). The QFT of the $h_{ \pm}$OPS split parts, with respect to two linearly independent unit quaternions $f, g$, of a quaternion module function $h \in L^{2}\left(\mathbb{R}^{2}, \mathbb{H}\right)$ have the complex forms

$$
\mathscr{F}_{ \pm}^{f, g}\{h\}=\int_{\mathbb{R}^{2}} h_{ \pm} e^{-g\left(x_{2} \omega_{2} \mp x_{1} \omega_{1}\right)} d^{2} x=\int_{\mathbb{R}^{2}} e^{-f\left(x_{1} \omega_{1} \mp x_{2} \omega_{2}\right)} h_{ \pm} d^{2} x
$$

The geometric interpretation of the integrand $e^{-f x_{1} \omega_{1}} h(\boldsymbol{x}) e^{-g x_{2} \omega_{2}}$ of the $\mathrm{QFT}^{f, g}$ in (17) is: The integrand means to locally rotate by the phase angle $-\left(x_{1} \omega_{1}+x_{2} \omega_{2}\right)$ in the $q_{-}$plane, and by phase angle $-\left(x_{1} \omega_{1}-x_{2} \omega_{2}\right)=x_{2} \omega_{2}-x_{1} \omega_{1}$ in the $q_{+}$plane.

The phase angle OPS-QFT with a straight forward two phase angle interpretation is

$$
\mathscr{F}_{D}^{f, g}\{h\}(\omega)=\int_{\mathbb{R}^{2}} e^{-f \frac{1}{2}\left(x_{1} \omega_{1}+x_{2} \omega_{2}\right)} h(\boldsymbol{x}) e^{-g \frac{1}{2}\left(x_{1} \omega_{1}-x_{2} \omega_{2}\right)} d^{2} \boldsymbol{x} .
$$

where again $h \in L^{1}\left(\mathbb{R}^{2}, \mathbb{H}\right), d^{2} \boldsymbol{x}=d x_{1} d x_{2}$ and $\boldsymbol{x}, \boldsymbol{\omega} \in \mathbb{R}^{2}$. Its inverse is given by

$$
h(\boldsymbol{x})=\frac{1}{(2 \pi)^{2}} \int_{\mathbb{R}^{2}} e^{f \frac{1}{2}\left(x_{1} \omega_{1}+x_{2} \omega_{2}\right)} \mathscr{F}_{D}^{f, g}\{h\}(\omega) e^{g \frac{1}{2}\left(x_{1} \omega_{1}-x_{2} \omega_{2}\right)} d^{2} \omega .
$$

The geometric interpretation of the integrand of (21) is a local phase rotation by angle $-\left(x_{1} \omega_{1}+x_{2} \omega_{2}\right) / 2-\left(x_{1} \omega_{1}-\right.$ $\left.x_{2} \omega_{2}\right) / 2=-x_{1} \omega_{1}$ in the $q$ - plane, and a second local phase rotation by angle $-\left(x_{1} \omega_{1}+x_{2} \omega_{2}\right) / 2+\left(x_{1} \omega_{1}-x_{2} \omega_{2}\right) / 2=$ $-x_{2} \omega_{2}$ in the $q_{+}$plane.

If we apply the OPS $^{f, g}$ split to (21) we obtain the following two parts

$$
\mathscr{F}_{D+}^{f, g}\{h\}=\int_{\mathbb{R}^{2}} h_{+} e^{+g x_{2} \omega_{2}} d^{2} x=\int_{\mathbb{R}^{2}} e^{-f x_{2} \omega_{2}} h_{+} d^{2} x, \quad \mathscr{F}_{D-}^{f, g}\{h\}=\int_{\mathbb{R}^{2}} h_{-} e^{-g x_{1} \omega_{1}} d^{2} x=\int_{\mathbb{R}^{2}} e^{-f x_{1} \omega_{1}} h_{-} d^{2} x .
$$

The OPS with respect to, e.g., $f=g=i$ gives

$$
q_{ \pm}=\frac{1}{2}(q \pm i q i), \quad q_{+}=q_{j} j+q_{k} k=\left(q_{j}+q_{k} i\right) j, \quad q_{-}=q_{r}+q_{i} i,
$$

where the $q_{+}$plane is two-dimensional and manifestly orthogonal to the 2D $q$ - plane. The above corresponds to the simplex/perplex split of [2]. $e^{\alpha f} q e^{\beta f}$ means a rotation by angle $\alpha+\beta$ in the $q$ - plane followed by a rotation by angle $\alpha-\beta$ in the orthogonal $q_{+}$plane.

A variant of the OPS-QFT with $g=f$ is therefore

$$
\mathscr{F}^{f, f}\{h\}(\omega)=\int_{\mathbb{R}^{2}} e^{-f x_{1} \omega_{1}} h(\boldsymbol{x}) e^{-f x_{2} \omega_{2}} d^{2} \boldsymbol{x},
$$

where $h \in L^{1}\left(\mathbb{R}^{2}, \mathbb{H}\right), d^{2} \boldsymbol{x}=d x_{1} d x_{2}$ and $\boldsymbol{x}, \omega \in \mathbb{R}^{2}$. The immediate geometric interpretation is that the integrand $e^{-f x_{1} \omega_{1}} h(\boldsymbol{x}) e^{-f x_{2} \omega_{2}}$ leads to a local phase roation by angle $-\left(x_{1} \omega_{1}+x_{2} \omega_{2}\right)$ in the $q$ - plane combined with a second local phase rotation by angle $x_{2} \omega_{2}-x_{1} \omega_{1}$ in the $q_{+}$plane. The inverse transform is given by

$$
h(\boldsymbol{x})=\frac{1}{(2 \pi)^{2}} \int_{\mathbb{R}^{2}} e^{f x_{1} \omega_{1}} \mathscr{F} f, f\{h\}(\omega) e^{f x_{2} \omega_{2}} d^{2} \omega,
$$

The phase angle OPS-QFT, using $g=f$, with a straight forward two phase angle interpretation is

$$
\mathscr{F}_{D}^{f, f}\{h\}(\omega)=\int_{\mathbb{R}^{2}} e^{-f \frac{1}{2}\left(x_{1} \omega_{1}+x_{2} \omega_{2}\right)} h(\boldsymbol{x}) e^{-f \frac{1}{2}\left(x_{1} \omega_{1}-x_{2} \omega_{2}\right)} d^{2} \boldsymbol{x} .
$$


where again $h \in L^{1}\left(\mathbb{R}^{2}, \mathbb{H}\right), d^{2} \boldsymbol{x}=d x_{1} d x_{2}$ and $\boldsymbol{x}, \omega \in \mathbb{R}^{2}$. Its inverse is given by

$$
h(\boldsymbol{x})=\frac{1}{(2 \pi)^{2}} \int_{\mathbb{R}^{2}} e^{f \frac{1}{2}\left(x_{1} \omega_{1}+x_{2} \omega_{2}\right)} \mathscr{F}_{D}^{f, f}\{h\}(\omega) e^{f \frac{1}{2}\left(x_{1} \omega_{1}-x_{2} \omega_{2}\right)} d^{2} \omega .
$$

The geometric interpretation of the integrand of 27 is a local phase rotation by angle $-\left(x_{1} \omega_{1}+x_{2} \omega_{2}\right) / 2-\left(x_{1} \omega_{1}-\right.$ $\left.x_{2} \omega_{2}\right) / 2=-x_{1} \omega_{1}$ in the $q$ - plane, and a second local phase rotation by angle $-\left(x_{1} \omega_{1}+x_{2} \omega_{2}\right) / 2+\left(x_{1} \omega_{1}-x_{2} \omega_{2}\right) / 2=$ $-x_{2} \omega_{2}$ in the $q_{+}$plane.

If we apply the OPS ${ }^{f, f}$ split to (27) we obtain the following two parts

$$
\mathscr{F}_{D+}^{f, f}\{h\}=\int_{\mathbb{R}^{2}} h_{+} e^{+f x_{2} \omega_{2}} d^{2} x=\int_{\mathbb{R}^{2}} e^{-f x_{2} \omega_{2}} h_{+} d^{2} x, \quad \mathscr{F}_{D-}^{f, f}\{h\}=\int_{\mathbb{R}^{2}} h_{-} e^{-f x_{1} \omega_{1}} d^{2} x=\int_{\mathbb{R}^{2}} e^{-f x_{1} \omega_{1}} h_{-} d^{2} x .
$$

The general OPS-QFT involving quaternion conjugation is defined as

$$
\mathscr{F}_{c}^{g, f}\{h\}(\omega)=\int_{\mathbb{R}^{2}} e^{-g x_{1} \omega_{1}} \widetilde{h(\boldsymbol{x})} e^{-f x_{2} \omega_{2}} d^{2} \boldsymbol{x},
$$

where $h \in L^{1}\left(\mathbb{R}^{2}, \mathbb{H}\right), d^{2} \boldsymbol{x}=d x_{1} d x_{2}$ and $\boldsymbol{x}, \omega \in \mathbb{R}^{2}$. The inverse is given by

$$
h(\boldsymbol{x})=\frac{1}{(2 \pi)^{2}} \int_{\mathbb{R}^{2}} e^{-f x_{2} \omega_{2}} \widetilde{\mathscr{F}_{c}^{g, f}\{h\}}(\omega) e^{-g x_{1} \omega_{1}} d^{2} \omega,
$$

This approach results in the following OPS theorem.

Theorem 3 (OPS-QFT $\mathscr{F}_{c}^{g, f}$ of $\left.h_{ \pm}\right)$. The OPS-QFT $\mathscr{F}_{c}^{g, f}$ (30) of the $h_{ \pm}=\frac{1}{2}(h \pm f h g)$ OPS split parts, with respect to two linearly independent unit quaternions $f, g$, of a quaternion module function $h \in L^{2}\left(\mathbb{R}^{2}, \mathbb{H}\right)$ have the complex forms

$$
\mathscr{F}_{c, \pm}^{g, f}\{h\}=\int_{\mathbb{R}^{2}} \widetilde{h_{ \pm}} e^{-f\left(x_{2} \omega_{2} \mp x_{1} \omega_{1}\right)} d^{2} x=\int_{\mathbb{R}^{2}} e^{-g\left(x_{1} \omega_{1} \mp x_{2} \omega_{2}\right)} \widetilde{h_{ \pm}} d^{2} x .
$$

The variant of the general OPS-QFT involving quaternion conjugation, using $g=f$, is defined as

$$
\mathscr{F}_{c}^{f, f}\{h\}(\omega)=\int_{\mathbb{R}^{2}} e^{-f x_{1} \omega_{1}} \widetilde{h(\boldsymbol{x})} e^{-f x_{2} \omega_{2}} d^{2} \boldsymbol{x},
$$

where $h \in L^{1}\left(\mathbb{R}^{2}, \mathbb{H}\right), d^{2} \boldsymbol{x}=d x_{1} d x_{2}$ and $\boldsymbol{x}, \omega \in \mathbb{R}^{2}$. The inverse is given by

$$
h(\boldsymbol{x})=\frac{1}{(2 \pi)^{2}} \int_{\mathbb{R}^{2}} e^{-f x_{2} \omega_{2} \mathscr{F}_{c}} \widetilde{f f}\{h\}(\omega) e^{-f x_{1} \omega_{1}} d^{2} \omega . .
$$

This results in the following OPS theorem.

Theorem 4 (OPS-QFT $\mathscr{F}_{c}^{f, f}$ of $h_{ \pm}$). The OPS-QFT $\mathscr{F}_{c}^{f, f}$ (33) of the $h_{ \pm}=\frac{1}{2}(h \pm f h f)$ OPS split parts, with respect to the unit quaternion $f$, of a quaternion module function $h \in L^{2}\left(\mathbb{R}^{2}, \mathbb{H}\right)$ have has complex forms

$$
\mathscr{F} c, \pm\{h\}=\int_{\mathbb{R}^{2}} \widetilde{h_{ \pm}} e^{-f\left(x_{2} \omega_{2} \mp x_{1} \omega_{1}\right)} d^{2} x=\int_{\mathbb{R}^{2}} e^{-f\left(x_{1} \omega_{1} \mp x_{2} \omega_{2}\right)} \widetilde{h_{ \pm}} d^{2} x .
$$

\section{ACKNOWLEDGMENTS}

Soli deo gloria. I do thank my dear family, S. Sangwine, W. Sprössig and K. Gürlebeck.

\section{REFERENCES}

1. H. S. M. Coxeter, Quaternions and Reflections, The American Mathematical Monthly, 53(3) (Mar., 1946), $136-146$.

2. T. A. Ell, Quaternionic-Fourier Transform for Analysis of Two-dimensional Linear Time-Invariant Partial Differential Systems. in Proceedings of the 32nd IEEE Conference on Decision and Control, December 15-17, 2 (1993), 1830-1841.

3. E. Hitzer, Quaternion Fourier Transform on Quaternion Fields and Generalizations, AACA, 17 (2007), 497-517.

4. E. Hitzer, Directional Uncertainty Principle for Quaternion Fourier Transforms, AACA, 20(2) (2010), $271-284$.

5. S. J. Sangwine, Fourier transf. of colour images using quat., or hyperc., numbers, El. Lett., 32(21) (1996), 1979-1980.

6. E. Hitzer, S.J. Sangwine, The orthogonal planes split of quaternions, in K. Guerlebeck (ed.), El. Proc. of ICCA9, Weimar, 2011. 